\title{
Detección de Fallas en el Aislamiento en las Chapas Del Estator de Máquinas Eléctricas Rotativas
}

\author{
Luisa Salazar ${ }^{1, *}$, Flavio Quizhpi ${ }^{2}$, José Manuel Aller $^{3}$, Alexander Bueno ${ }^{4}$, Rodney Reyna ${ }^{5}$
}

\section{Resumen}

En este trabajo se comparan dos metodologías para la detección de cortocircuitos y fallas entre chapas de núcleos magnéticos, para lo cual se realizaron los ensayos sobre las chapas que conforman el estator de un motor de inducción, con el objetivo de determinar el método de detección más eficaz. Los métodos sometidos a comparación son: Método de la prueba de aislamiento del laminado del núcleo del estator, recomendado por el estándar IEEE-56 Guide for Insulation Maintenance of Large Alternating - Current Rotating Machinery (10000 KVA and Larger) y el método de variaciones de flujo, el cual permite mediante dos electroimanes o bobinas (sensora y patrón) sobre las chapas, determinar las variaciones en magnitud y fase de la corriente que circula por las mismas, ubicando los puntos de falla.

Palabras clave: Aislación, Bobina, Diente, Laminado, Núcleo Magnético.

\begin{abstract}
This paper compares two methods for detecting short circuits and faults between the plates of magnetic cores, for which the tests were performed on sheet metal forming the stator of an induction motor, in order to determine the method of more effective screening. The methods under comparison are: Method of isolation test laminated stator core, recommended by the IEEE-56 Guide for Insulation Maintenance of Large Alternating - Current Rotating Machinery (10000 kVA and Larger) and flow variations method, which allows using two electromagnets or coils (sensor and pattern) on the plates, determine variations in magnitude and phase of the current flowing through them, locating points of failure.
\end{abstract}

Keywords: Thermal imager, Tooth, Rolling, Roll.

\footnotetext{
1,* Máster en Sistemas Eléctricos de Potencia, Ingeniero Electricista, Docente del Departamento de Tecnología Industrial, Universidad Simón Bolívar, Caracas, Venezuela. Autor para correspondencia: luisalazar@usb.ve ${ }^{2}$ Ingeniero Electrónico, Estudiante de Doctorado en Ingeniería Eléctrica - Universidad Simón Bolívar, Caracas, Jefe del Área de Investigación de las Carreras de Ingenierías Eléctrica y Electrónica, Universidad Politécnica Salesiana, sede Cuenca.

${ }^{3}$ Profesor Especialidad Física. Magíster Scientiarum en Física de los Ensayos no Destructivos, Docente Universidad Simón Bolivar, Caracas, Venezuela.

${ }^{4}$ Doctor en Sistemas de Energía Eléctrica, Magister Scientarum en Ingeniería Eléctrica, Ingeniero Electricista. Director del GSIEP - Universidad Simón Bolívar, Caracas, Venezuela.

${ }^{5}$ Doctor en Ciencias de la Ingeniería, Magister Scientarum en Ingeniería Eléctrica, Ingeniero Electricista, Coordinador de la Carrera de Ingeniería Eléctrica de la Universidad Simón Bolívar, Caracas, Venezuela
}

Recibido: 04 - Febrero - 2012; Aprobado tras revisión: 18 - Mayo - 2012.

Forma sugerida de citación: Salazar, L., et al. (2012). "Detección de Fallas en el Aislamiento en las Chapas del Estator de Máquinas Eléctricas Rotativas". INGENIUS. Nㄱ, (enero/junio). pp 11-20. ISSN: 1390-650X 


\section{Introducción}

En la operación de sistemas de potencia existe un uso extendido de equipos para la conversión de energía (Transformadores de potencia y máquinas eléctricas rotativas), tradicionalmente estos equipos trabajan en base a los principios de la teoría electromagnética. Estos equipos en constitución tienen núcleos ferromagnéticos los cuales se estructuran a partir de láminas, eléctricamente aisladas entre sí, con la finalidad de disminuir las pérdidas por inducción de corrientes parásitas.

Este aislamiento entre las láminas o chapas de los núcleos magnéticos está constituido por baños galvánicos o en caso de sistemas más antiguos por barnices, capas de óxidos, etc. Este aislamiento puede fallar ocasionando contactos físicos entre las láminas. Por otra parte, los núcleos magnéticos están sometidos a importantes intensidades de campos magnéticos, al existir fallas entre las chapas se incrementan las corrientes parásitas ocasionando puntos calientes que pueden llevar a elevar la temperatura, ocasionando incremento en las pérdidas del convertidor, y deterioro del aislamiento de los conductores cercanos al sitio de falla.

El objetivo de esta investigación se centra en comparar dos métodos, uno sustentado por la norma IEEE-56 y el otro basado en el trabajo en [1]. Estos métodos permiten determinar puntos de falla en el laminado del estator de las máquinas eléctricas rotativas. El primer método compara el gradiente de temperatura, en tanto que el segundo método propuesto, considera variación de las intensidades, en magnitud y fase, de las corrientes que alimentan a bobinas (patrón y sensora), el análisis de los resultados del segundo método permite determinar puntos de falla directamente sin la necesidad de energizar el núcleo a flujo nominal. Al comparar los procedimientos y resultados obtenidos se determina la eficacia de ambos métodos para detectar fallas en el estator y la factibilidad de implementación como método de diagnóstico en campo.

\section{Marco teórico}

\subsection{Método 1}

De forma axial al estator de la máqquina de inducción se colocó una bobina, a través de la cual se inyectó corriente alterna para lograr establecer un flujo muy cercano al codo de saturación del convertidor. La bobina se colocó en forma axial, tal como se muestra en la Figura 1, para que el flujo magnético se mantenga dentro del laminado estatórico y así los puntos donde existen fallas, entre chapas, queden sometidos a la variación de flujo circulante por el laminado. En los puntos de falla se producen calentamientos como resultado del mayor gradiente de temperatura respecto a los diferentes puntos del laminado.

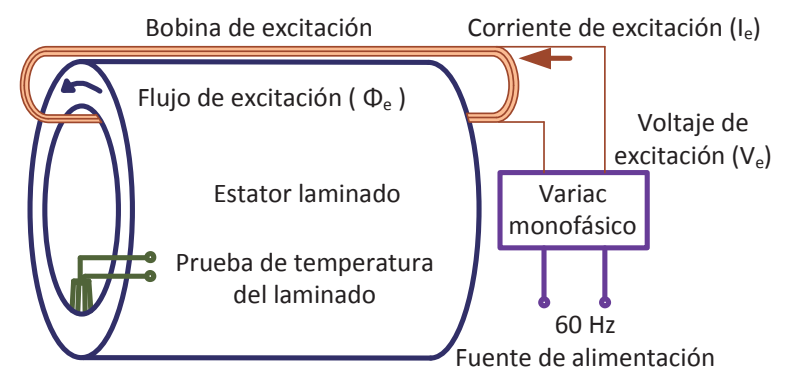

Figura 1. Diagrama de montaje Método 1

La bobina utilizada se diseñó a partir de una densidad de campo magnético típica y la dimensión del núcleo magnético del estator, mediante las expresiones:

$$
\phi=B A
$$




$$
\begin{gathered}
E=4,44 \phi f N \\
H=\frac{N_{i}}{l} \\
B=\mu_{0} \mu_{r} H
\end{gathered}
$$

Y los datos de la máquina son:

\section{SIEMENS}

$0-91056$

3 MOT 1LC4 228 - 4AA10 - Z 225 M

UC 0701/014720201/MB3Th C.F

\begin{tabular}{ccccccc}
\hline$V$ & $H z$ & $A$ & $k W$ & $\operatorname{Cos} \phi$ & $1 / \min$ & $I_{p}$ \\
\hline $230 \Delta$ & 50 & 172 & 55 & 0,86 & 1475 & 65 \\
\hline $400 Y$ & & 99 & & & & \\
\hline $400 Y$ & 60 & 97 & 63 & 0,87 & 1775 & \\
\hline
\end{tabular}

IEC / EN 60034 Gew/w. 330 kg

$50 \mathrm{~Hz}$

\begin{tabular}{ccc}
\hline Conexión & Tensión & Corriente \\
\hline$\Delta$ & $220-240 V$ & $176-170 A$ \\
\hline$Y$ & $380-420 V$ & $102-97 A$ \\
\hline
\end{tabular}

Considerando del material del estator:

$$
B=0,5 W b / m^{2}
$$

Y los datos de la máquina son:

Diámetro Exterior laminado estator, $D_{\text {ext }}=332 \mathrm{~mm}$

Diámetro Interior laminado estator, $D_{\text {int }}=232 \mathrm{~mm}$

Longitud del Núcleo, $l_{\text {nuc }}=430 \mathrm{~mm}$

La forma y las dimensiones son mostradas en la Figura 2

Sustituyendo en 1, se tiene que:

$$
\phi=(0,5)(0,0258)=0,0129 \mathrm{~Wb}
$$

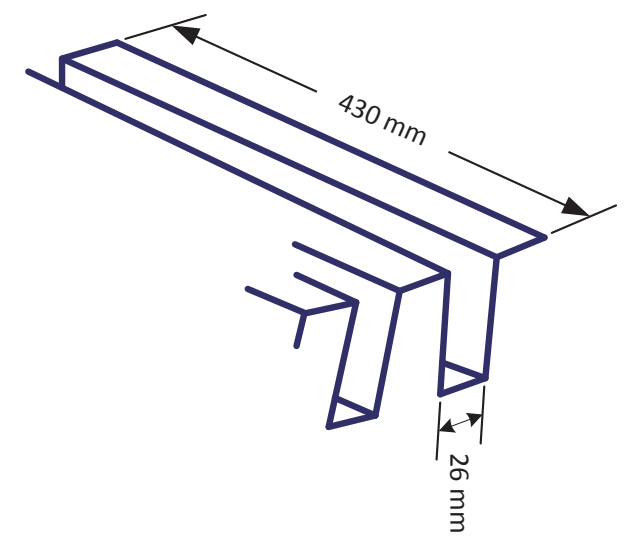

Figura 2. Dimensiones del Núcleo del Estator

Sustituyendo en 2, se tiene que:

$$
E=(4,44)(0,0129)(60)(17)=58,42 \mathrm{~V}
$$

La longitud de la corona estatórica según el estándar IEEE-56 se calcula a través de:

$$
l=\frac{\left(D_{e x t}+D_{i n t}\right) \pi}{2}
$$

$$
l=0,9174 m
$$

Tomando en cuenta que $\mu_{r}=800$ para núcleos de hierro laminado y con el valor de la Ecuación 5, la intensidad de campo magnético se obtiene de la Ecuación 4, que es la corriente de trabajo $\left(i_{\text {alim }}\right)$.

$$
H=\frac{0,5}{4 \pi 10^{-7} 800}=497,35 \mathrm{~A} / \mathrm{m}
$$

$$
i_{\text {alim }}=\frac{H l}{N}=497,35 \frac{0,97}{17}=28,37 \mathrm{~A}
$$

Debido a limitaciones prácticas (corriente nominal del transformador de relación variable. $V_{\text {prim }}=240 \mathrm{~V}, V_{\text {regulable }}=0-24 \mathrm{~V}$, $I_{\text {nom }}=18 \mathrm{Amp}$ en el laboratorio, la corriente máxima a la cual se puede trabajar, teniendo control sobre la tensión de alimentación y 
considerando una sobrecarga, es de $20 A$; por lo que, se ajustó el número de vueltas de la bobina de acuerdo a esta corriente y de la Ecuación 4 se tiene un bobinado de 24 vueltas. Se hace circular la corriente de tal forma que se logra calentar el núcleo.

En el núcleo se introdujeron puntos de falla entre láminas, con la finalidad de tener localizados posibles puntos calientes. Los cortocircuitos (Figura 3) se provocaron con un anillo, tipo espira sombra, de cobre en la parte frontal y posterior del diente 1 .

El sistema de acuerdo al montaje de la Figura 1 se energizó para calentar el laminado y tomar lecturas de temperatura, con una cámara termográfica Marca Fluke, modelo Ti25, ajustada a una emisividad de 0,88 que corresponde al material de acero laminado. Se cubren los cuarenta y ocho dientes laminados, dividiendo en secciones, con el objeto de observar gradientes de temperatura en los diferentes puntos del laminado. Se consideran puntos de falla donde exista mayor gradiente de temperatura.

\subsection{Método 2}

Consiste en detectar variaciones de flujo en los puntos de falla del laminado estatórico, respecto a un patrón sin fallas. El método desarrollado en [1] y [2], detectan variaciones de flujo a partir de un flujo único circulante en el paquete laminado. Para detectar la falla de desliza un electro imán sobre las chapas del estator y se registran la corriente (magnitud y fase) y se compara con un patrón de laminado sano, al encontrar puntos donde existan fallas existirá un cambio en la corriente (magnitud y fase).

Para aplicación del ensayo práctico se hizo una variante, la cual consistió en utilizar dos

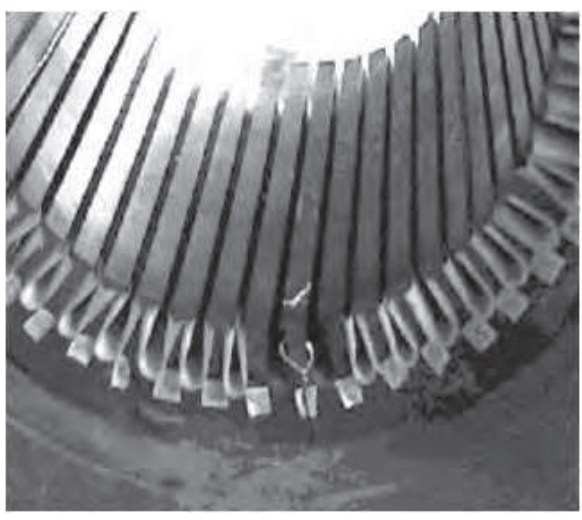

(a) Vista Frontal

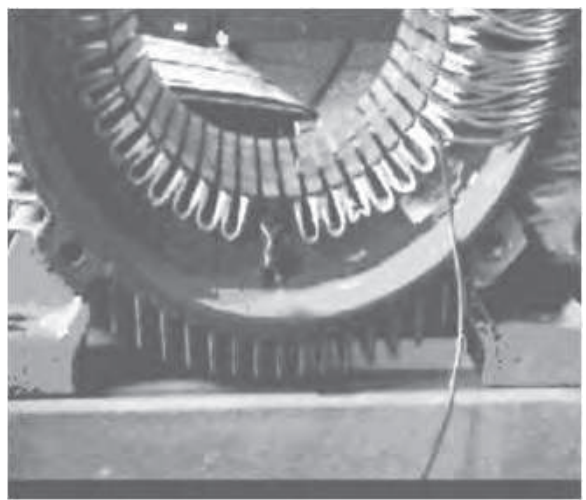

(b) Vista Posterior

Figura 3. Disposición de la Falla en el diente 1

bobinas de idénticas características (forma del núcleo, tamaño, número de espiras) mostrados en la Figura 4, con la idea de cerrar el circuito magnético con parte de láminas del paquete de chapas del estator, tal como se presenta en la Figura 5. Cada una de las bobinas, es excitada con la misma fuente de tensión (en paralelo), la primera bobina (patrón) se mantiene fija y la segunda (sensora) se desplaza sobre los dientes del circuito magnético del estator. Si el laminado del estator no presenta falla, los registros de corriente deben ser iguales, en caso contrario se tendrán diferencias y existirá un punto de falla, o uno posible, lo cual debe verificarse con inspección visual.

Las dimensiones del núcleo magnético de la bobina fija y de la que actúa como detectora de las variaciones de flujo son idénticas y para 

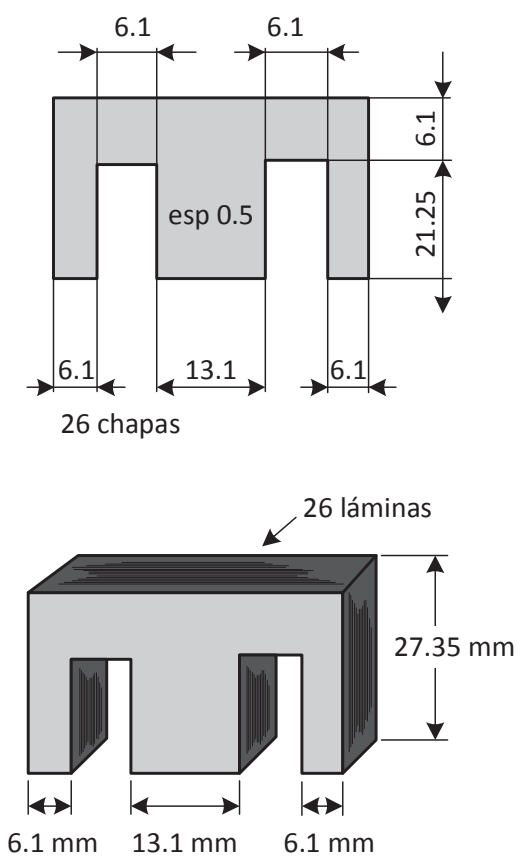

Figura 4. Dimensiones de las bobinas sensor y patrón

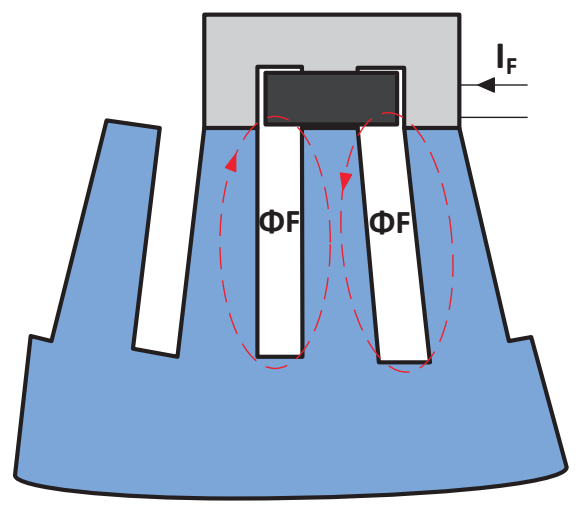

Figura 5. Recorrido de los flujos de las bobinas, sensor y patrón.

el dimensionamiento se toman en cuenta las siguientes consideraciones:

a.- El ancho de la columna central cubrirá el ancho del diente a evaluar, ésta característica hace que se limite el ensayo a un grupo de máquinas que tengan laminados de chapas con idénticos anchos de diente.

b.- El valor inicial para ubicar la corriente que genera el flujo en las bobinas de referencia y sensora se ve limitado por la característica térmica del conductor, porque sin el cierre del circuito magnético del núcleo de las bobinas, éstas se encuentran sin saturación (parte del núcleo es aire).

Para realizar el ensayo se utilizó el circuito mostrado en la Figura 6, en el cual se presenta el diagrama esquemático utilizado en el proceso. Como se observa las dos bobinas se alimentan con la misma fuente, manteniendo una bobina fija y la otra móvil se van tomando registros para evaluar todos los dientes del laminado.

Una vez que se tienen las bobinas para el ensayo, cada una se ubica en una sección de las ranuras, en puntos que se pueden considerar como referencia (sin falla). Para tomar la muestra se realiza la grabación de las formas de onda de corriente de las dos bobinas. En los puntos iniciales de referencia, sobre las dos bobinas existirán dos corrientes que se consideran corrientes de vacío.

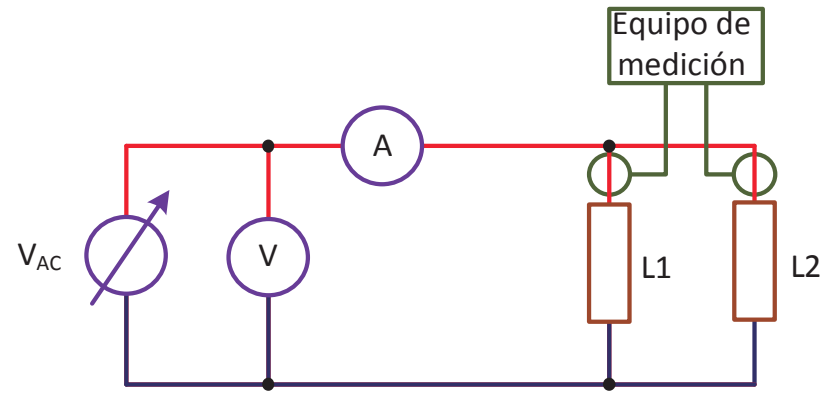

Figura 6. Circuito de montaje para el método 2

Posteriormente se fija una bobina como referencia para el ensayo (patrón) y la de sensado se desplazará a lo largo de los diferentes dientes del laminado estatórico. En el momento que se generen las fallas se observarán cambios de corriente en la bobina de sensado, debido a que en este punto se producen variaciones del camino magnético que deformarán los valores del flujo de vacío, estas deformaciones resultado de las variaciones del flujo primario producen cambios en las corrientes 
de vacío del punto de partida. Los cambios de la corriente son captados en los canales del osciloscopio que se usa como equipo de medición.

\section{Análisis de resultados}

Una vez revisados los registros obtenidos de la termografía del método 1 y las gráficas de corriente del método 2, para sustentar la validez del método propuesto se seleccionaron 3 dientes de los cuales dos presentan fallas, una provocada y otra propia del laminado, y uno es un diente sano. Los dientes objeto de estudio son el 1, 3 y 26 (Tabla 1).

Tabla 1. Dientes seleccionados para el análisis

\begin{tabular}{cl}
\hline $\mathbf{N}^{\circ}$ de Diente & Condición Operativa \\
\hline 1 & $\begin{array}{l}\text { Cortocircuito frontal y posterior } \\
\text { con una lámina }\end{array}$ \\
\hline 26 & Con Falla propia del laminado \\
\hline 3 & Sano \\
\hline
\end{tabular}

En la presentación de los resultados se consideran: la imagen de la cámara termográfica, el resultado de la comparación de las corrientes patrón y sensora (expresada en el comportamiento XY), en donde si no existe falla, las dos corrientes están en fase; y al existir falla se forma un lóbulo con diferencia de fase en el análisis XY.

\subsection{Resultados de diente con falla pro- vocada}

Se presenta, en la Figura 7a, la imagen térmica de la parte frontal del estator con las temperaturas de los diferentes puntos del diente 1. En la Figura 7b está el registro de corrientes de las bobinas patrón y sensora en la parte frontal del diente 1; colocada la bobina patrón en el punto inicial del diente 1 y la sensora en el punto de falla. La Figura 7c, presenta la im- agen térmica de la parte posterior del estator con las temperaturas del diente 1. La Figura $7 \mathrm{~d}$ presenta el registro de corrientes correspondientes a la patrón y sensora al punto de falla del diente 1 en la parte posterior.

De los resultados expuestos en las Figuras 7a y 7c se observa que las variaciones de temperatura en los extremos donde es forzada la falla la temperatura es considerablemente elevada con respecto a lo largo del laminado, los registros varían gradualmente hasta alcanzar el valor más alto teniendo en la parte frontal $38,7^{\circ} \mathrm{C}$ y en la parte posterior $39,1^{\circ} \mathrm{C}$ para promedios de temperatura de ambos laminados de $36,58^{\circ} \mathrm{C}$ y $38,06{ }^{\circ} \mathrm{C}$ con desviaciones con respecto al promedio de $3,9 \%$.

De los resultados gráficos expuestos en las Figuras 7b y 7d se observa que las corrientes presentan corrimiento de fase y diferencia de la amplitud de la bobina sensora con respecto a la patrón en los puntos de falla, en la Figura $7 d$ hay mayor desplazamiento de la onda de la bobina sensora pues la temperatura en el punto de falla es mayor que en el otro extremo.

\subsection{Resultados de diente con falla iden- tificada}

Identificado como punto de falla sobre el diente 26, la Figura 8a presenta la imagen termográfica del diente 26, y la Figura 8b presenta el registro de corrientes de bobina patrón y sensora sobre el punto de falla en diente 26.

De los resultados gráficos y de acuerdo a la referencia de lo expuesto para el diente 1, el comportamiento termográfico y de las corrientes determinan punto de falla, el registro de temperatura se presenta en forma gradual pero hay dos lecturas de temperatura $39^{\circ} \mathrm{C}$ y $39,6^{\circ} \mathrm{C}$; que de acuerdo a las lecturas presen- 


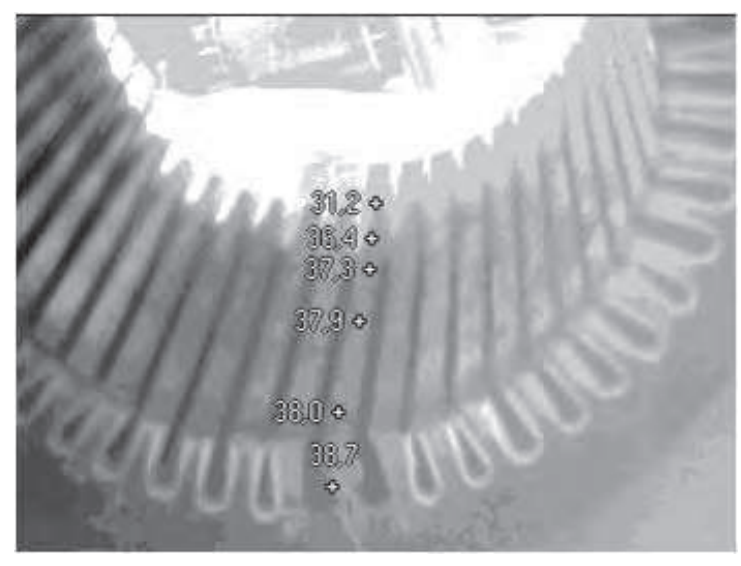

(a) Temperaturas diente 1, vista frontal

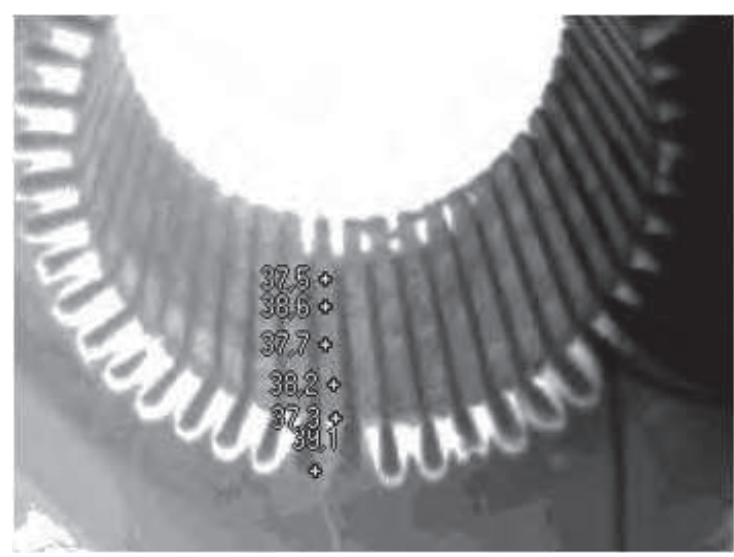

(c) Temperaturas diente 1, vista posterior

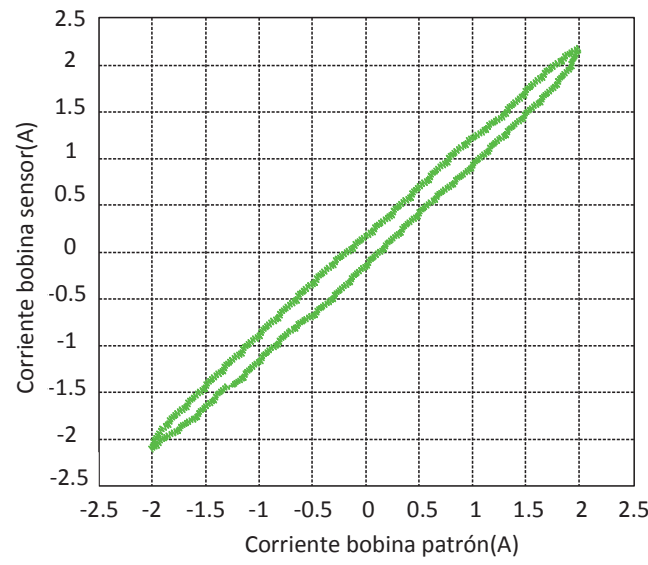

(b) Relación entre corriente patrón y sensora, diente 1 frontal

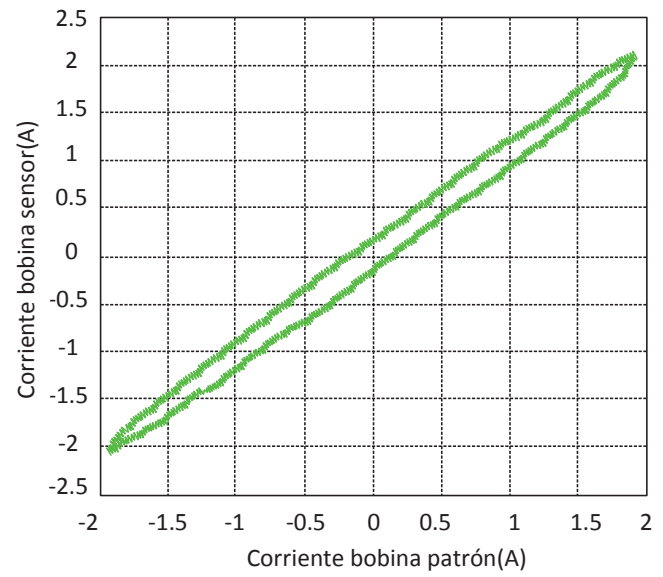

(d) Relación entre corriente patrón y sensora, diente 1 posterior

Figura 7. Resultados de diente con falla provocada

tadas nos indican el punto de falla. Esta afirmación es corroborada con la Figura 8b donde se observa desplazamiento de fase y variación de amplitud de la onda de corriente sensora respecto a la patrón.

\subsection{Resultados de diente identificado como sano}

Seleccionado un diente que presenta un comportamiento uniforme, tanto para la imagen termográfica como para el análisis de las corrientes de las bobinas sensora y patrón. En la figura 9.a se ve las temperaturas de los puntos de referencia del diente 3 en la parte frontal y en la Figura 9a el registro de corrientes de la bobina patrón y sensora.

De acuerdo a los resultados del registro termográfico las temperaturas tienen variaciones graduales en el orden promedio de $0,3{ }^{\circ} \mathrm{C}$ con una temperatura promedio $36,35^{\circ} \mathrm{C}$ a lo largo del laminado, por lo que el comportamiento de la temperatura es uniforme lo que indica que de acuerdo a las referencias de los resultados obtenidos en los dientes 1 y 26 el diente 3 no presenta falla y es considerado como sano, esto corroborado con el comportamiento de la onda de corriente de la bobina sensora, la cual no tiene variación de fase y amplitud con res- 


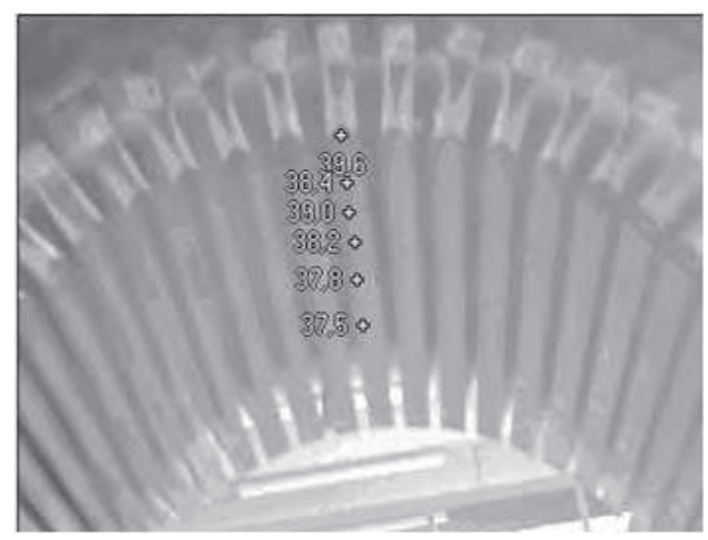

(a) Temperaturas diente 26, vista frontal

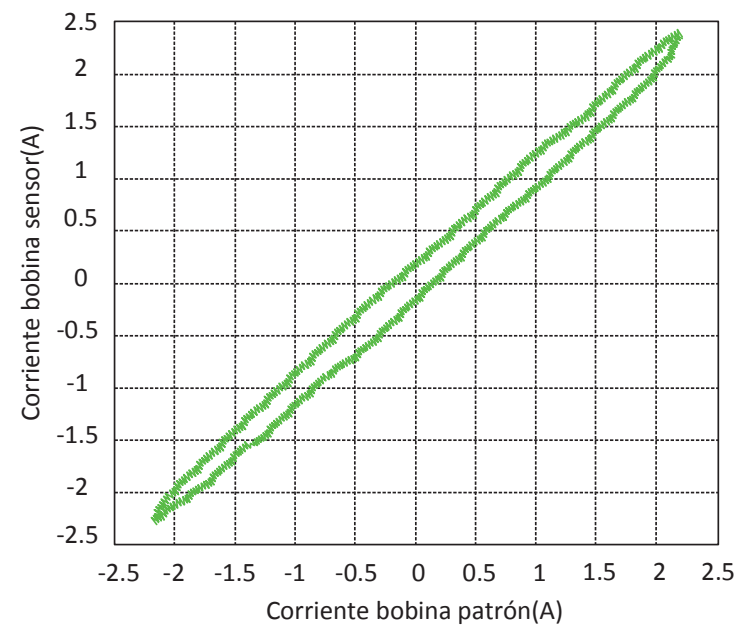

(b) Relación entre corriente patrón y sensora, diente 26 frontal

Figura 8. Resultados de diente con falla provocada

pecto onda de la bobina patrón, de acuerdo a lo observado en la Figura 9b.

\section{Recomendaciones}

El objetivo de lograr un método simple que permita verificar la presencia de falla de las láminas del estator de una máquina de inducción, inicialmente se demostró que es factible. Sin embargo se presentan recomendaciones que deberían implementarse para lograr que la aplicación del método sea eficaz.

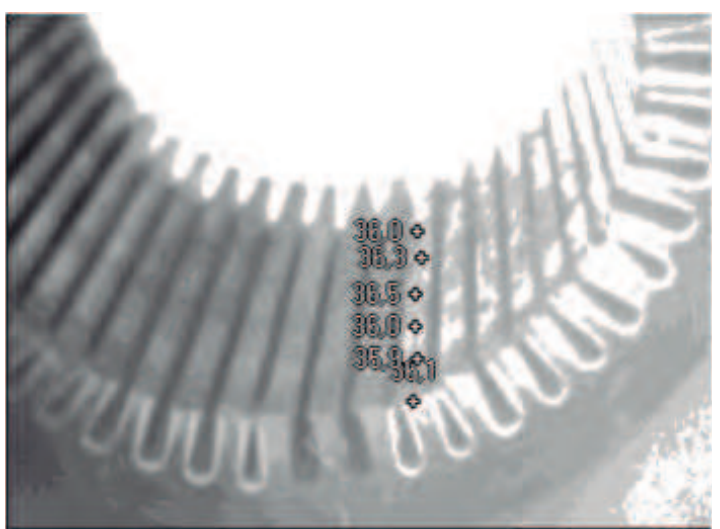

(a) Temperaturas diente 3, vista frontal

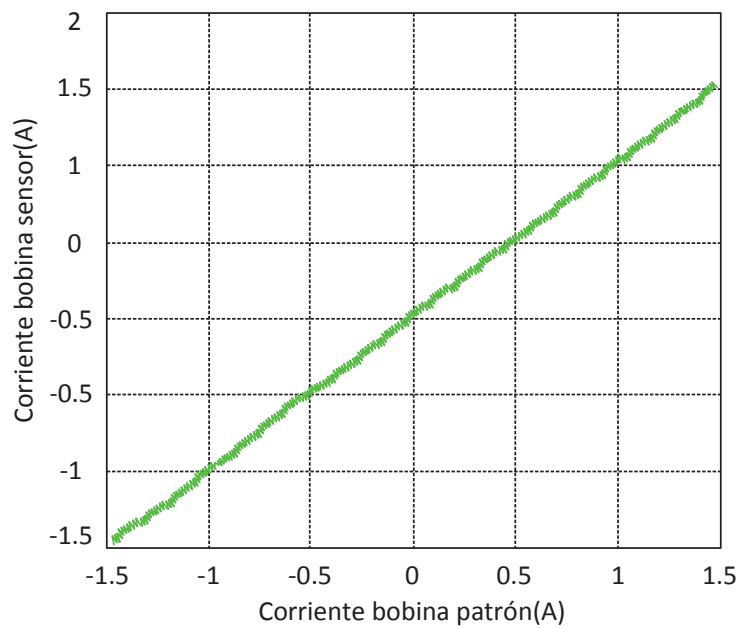

(b) Relación entre corriente patrón y sensora, diente 3 frontal

Figura 9. Resultados de diente con falla provocada

\section{Para el método 1}

Se debe tener condiciones ambientales constantes durante el ensayo. Si la toma de data de las temperaturas son realizadas con termómetros, buscar en lo posible barridos en espacios uniformes.

Al utilizar la cámara termográfica se tiene un espectro de temperaturas matriciales de la foto que se obtiene, por lo que, debería elaborarse un algoritmo para extraer la información del laminado del diente en particular para poder realizar el estudio y determinar los cambios de temperatura.

Con la data obtenida realizar un estudio 
del comportamiento del material usado en la construcción del laminado del estator con respecto al aislante entre los laminados que conforman el diente. En consecuencia determinar que en las variaciones de temperatura presentadas no interviene, significativamente, el tipo de aislante usado entre láminas.

Realizar un estudio de las pérdidas de potencia en puntos de variaciones significativas de temperatura por disipación de calor con objeto de determinar la reducción de la eficiencia del motor y permitir parametrizar la máquina a valores nominales.

\section{Respecto al método 2}

La superficie que conforma el laminado de los dientes presentan rugosidades que ocasionan errores, debido a que la bobina se separa de la superficie de contacto, bajo esta condición la lectura de corriente podría tener distorsiones, que no necesariamente signifique puntos de falla del aislamiento de las láminas del estator, por lo que se debe disponer de un mecanismo que permita que el deslizamiento de la bobina sensora se mantenga constante, sobre la superficie de los dientes a ser analizados.

Garantizar que el equipo de registro tenga la suficiente sensibilidad, de tal manera que, los registros sean en forma continua durante el ensayo sobre el diente, por lo que debería automatizarse la toma de datos.

\section{Conclusiones}

Se validó el método propuesto a través de una serie de comparaciones de los datos obtenidos en los dos métodos.

Por lo tanto la eficacia del método propuesto garantiza que en el $100 \%$ se detectan los puntos fallados.
El método propuesto indica la presencia de cortocircuitos entre las chapas, situación notoria en los puntos en los que se introdujeron fallas forzadas, que permitieron validar la eficacia del método.

No todos los puntos calientes registrados en el método uno representan fallas en el aislamiento de las chapas, en algunos casos se presenta un aumento en temperatura debido a la existencia de una falla en las cercanías, o es notorio un mayor calentamiento en las zonas cercanas a la región de la bobina usada para calentar el núcleo.

Del análisis de los datos de variación de temperatura se observan dientes que tienen cambios de temperatura, que podrían calificarse de significativos, pero luego de analizar con el método de variación de flujo, se concluye que los cambios de temperatura se deben a los efectos de calentamiento por conducción de los dientes que presentan falla (situación que se observa al ver que no existe variación de las amplitudes de corriente, método 2) cercanos al diente en estudio. Esta situación es evidente alrededor de los dientes en donde se tenían la falla forzada, observándose cambios de temperatura y significativos cambios en las amplitudes y fase de las corrientes que alimentan las bobinas sensor y la de referencia.

Se observa que en los dientes que están cercanos a la bobina de generación del flujo constante presentan mayores calentamientos, situación que se atribuye a que en esa región el flujo de dispersión, generado por la bobina, afecta al material cercano a la misma, en este caso los dientes cercanos.

El consumo de energía, en el método dos, es muy bajo (12 W-h) en los elementos sensores, si lo comparamos con el uno, en donde la demanda de energía para el calentamiento del 
núcleo es elevada $(800 W-h)$. Además este método resulta económico ya que la construcción de las bobinas tiene un precio estimado de $4 \$$ y el osciloscopio $3800 \$$, para un total de 3804 \$ en comparación con el método 1 donde se necesita la cámara termográfica la cual tiene un precio estimado de $20.000 \$$.

La presencia de magnetismo remanente en el núcleo no afecta la eficiencia del método dos, porque el sentido del campo magnético generado por las bobinas patrón y sensora, solo abarca una región, que si la comparamos con la longitud del paquete laminado del estator, es poco significativa.[3]

\section{Referencias}

[1] P. Zlatimir, "Inspection of stator cores in large machines with a low yoke induction method measurement and anlysis of interlamination short circuits," IEEE, vol. 16, pp. 81-86, 2002.

[2] L. Sang Bin, B. Gerald, G. Kliman, R. Manoj, and M. Shah, "Experimental study of inter - laminar core fault detection techniques based on low flux core excitation," IEEE, vol. 21, pp. 85-94, 2005.

[3] W. McDermid, H. Manitoba, and M. Winnipeg, "Guide for insulation maintenance of large alternating - current rotating machinery (10000 KVA and larger)," IEEE, vol. 9, pp. 7-15, 1993.

[4] L. Sang Bin, B. Gerald, G. Kliman, R. Manoj, M. Shah, N. Kutty, and L. Mark, "An iron core probe based inter laminar core fault detection technique for generator stator cores," IEEE, vol. 20, pp. 344-351, 2005. 